\title{
Author Correction: Non-coding RNAs participate in the regulatory network of CLDN4 via ceRNA mediated miRNA evasion
}

Yong-xi Song, Jing-xu Sun, Jun-hua Zhao, Yu-chong Yang, Jin-xin Shi, Zhong-hua Wu, Xiao-wan Chen, Peng Gao, Zhi-feng Miao \& Zhen-ning Wang

Correction to: Nature Communications https://doi.org/10.1038/s41467-017-00304-1, published online 18 August 2017

In the original version of this Article, the images in the Figure $2 c$ column labelled "CLDN4+ miR-3620-3p mut' were inadvertently replaced with duplicates from the Figure $2 c$ column labelled "CLDN4+". In addition, the Figure $2 \mathrm{f}$ column "CLDN4+ miR-3620-3p mut' was inadvertently labelled "CLDN4+ miR-3620-3p". The original and the corrected figures are presented below. 


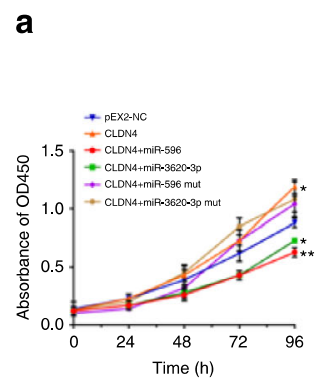

b
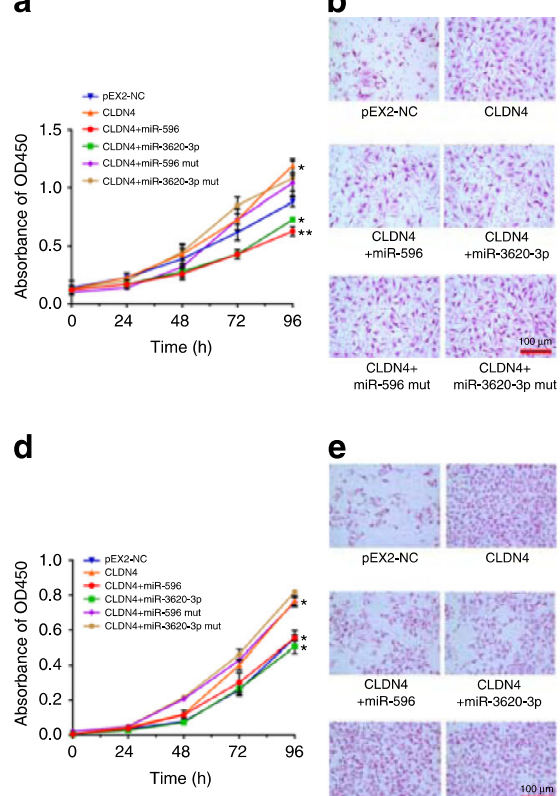

e
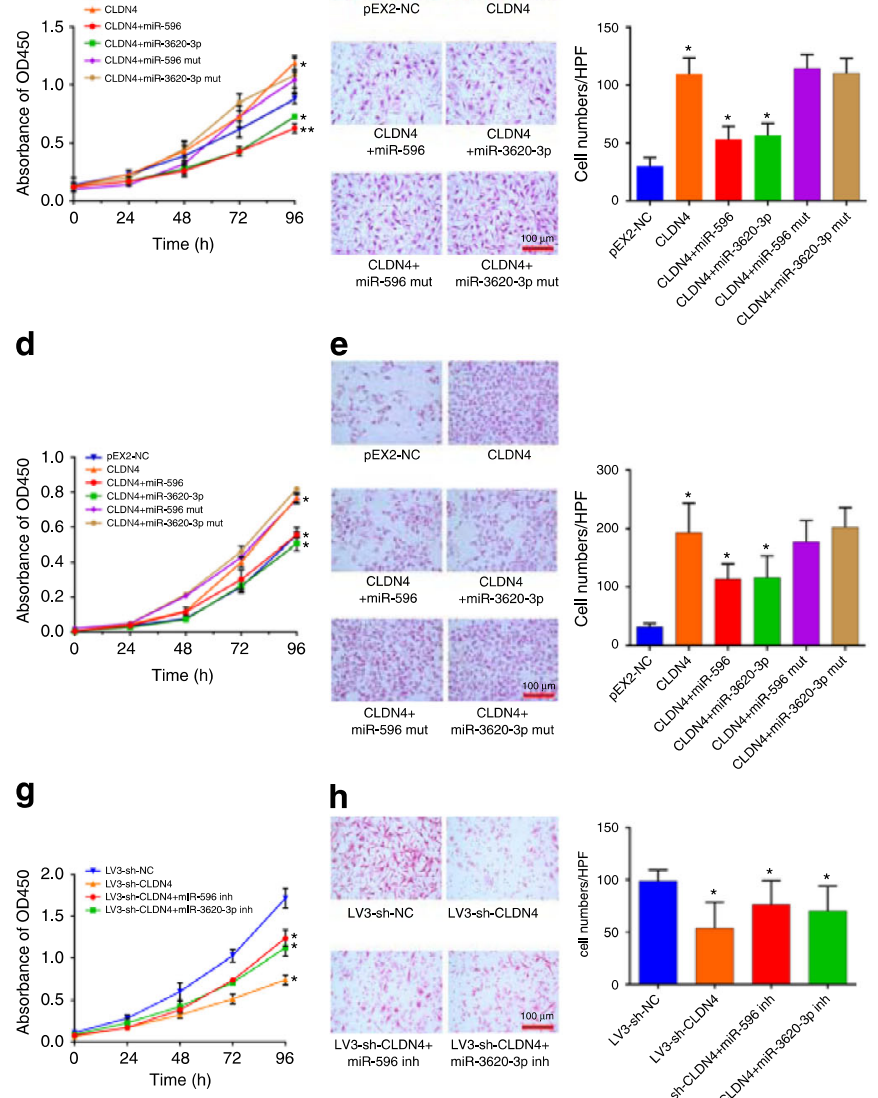

h
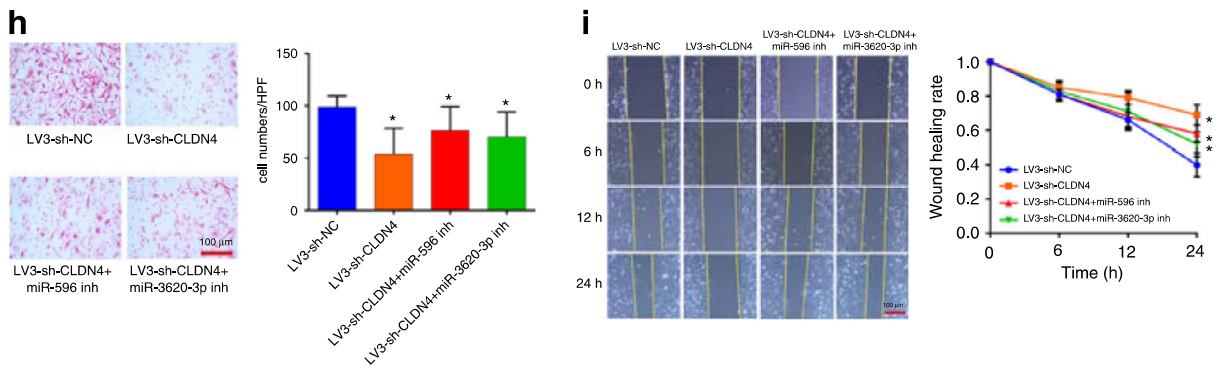

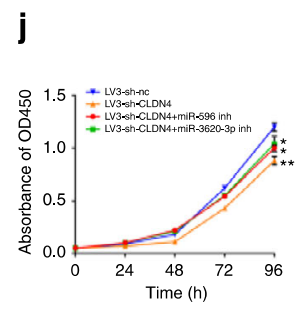

k

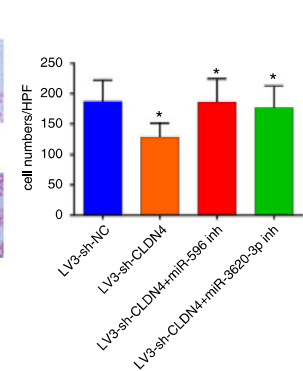

I
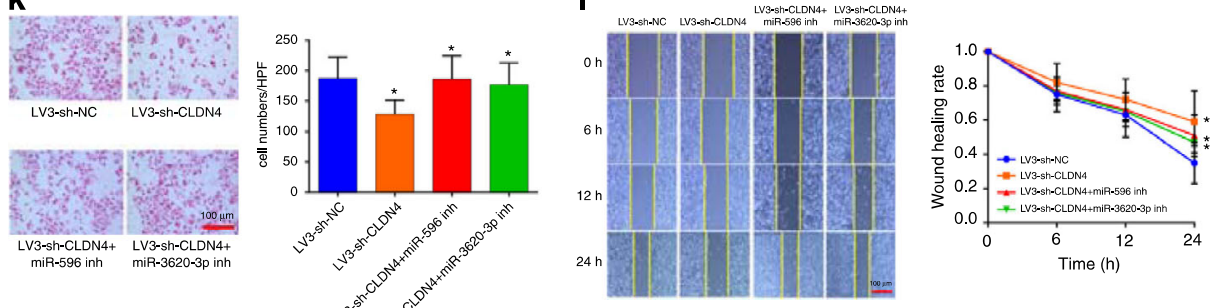
a

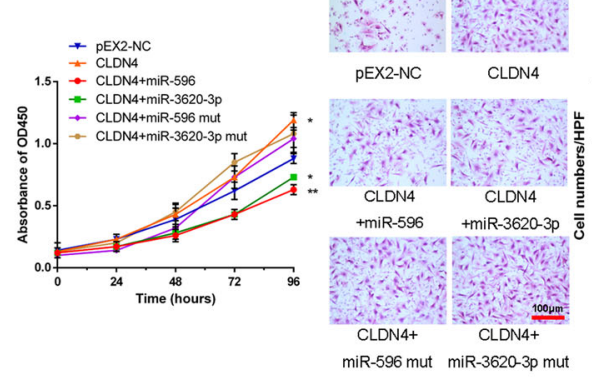

d

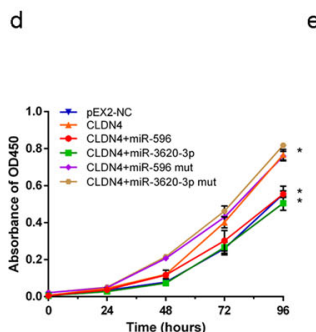

g
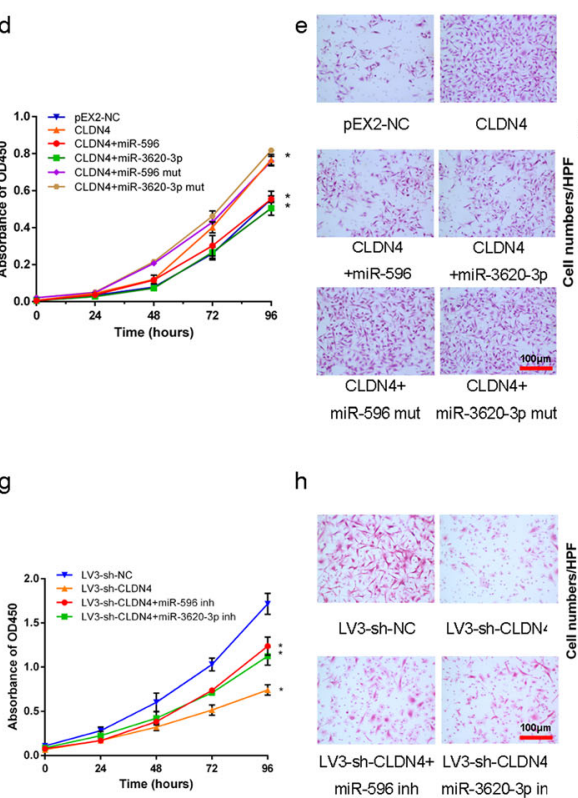

$\mathrm{h}$

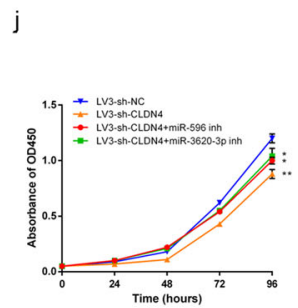

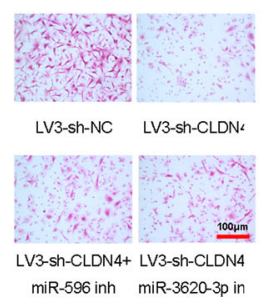

k

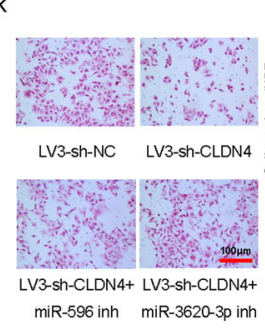

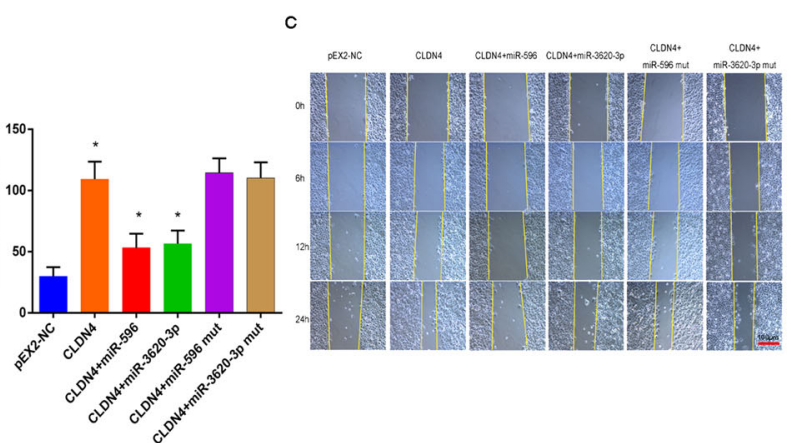

c
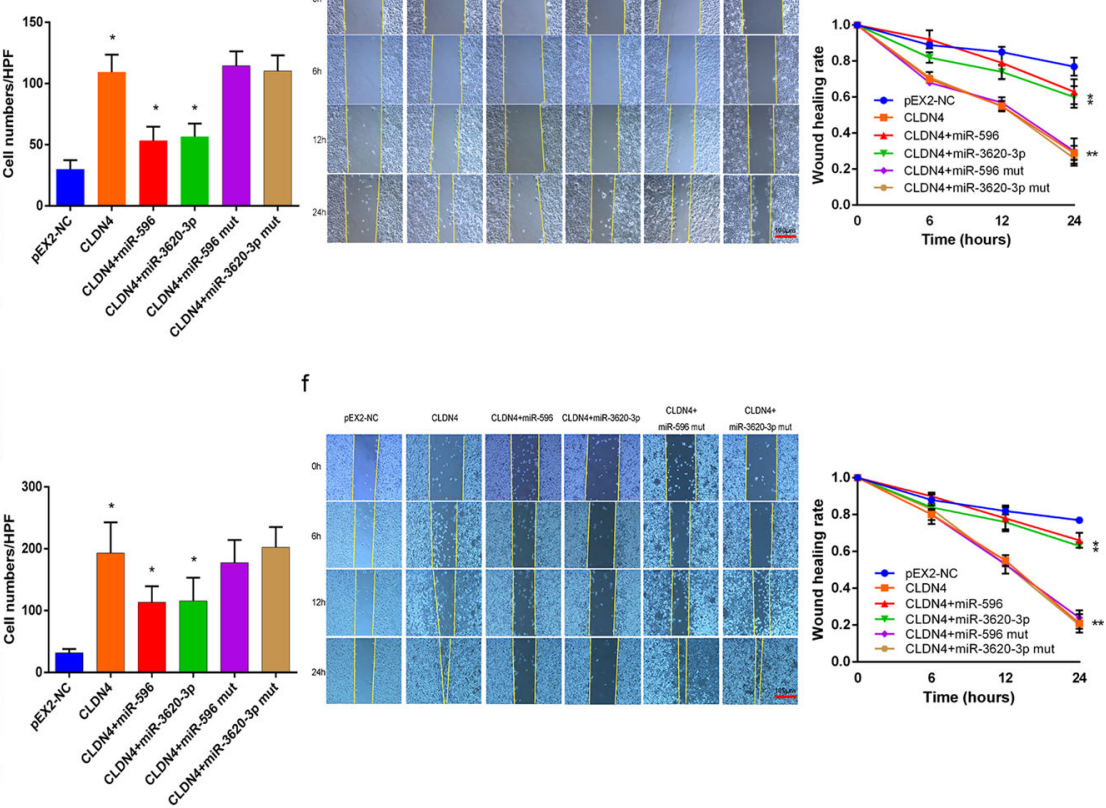
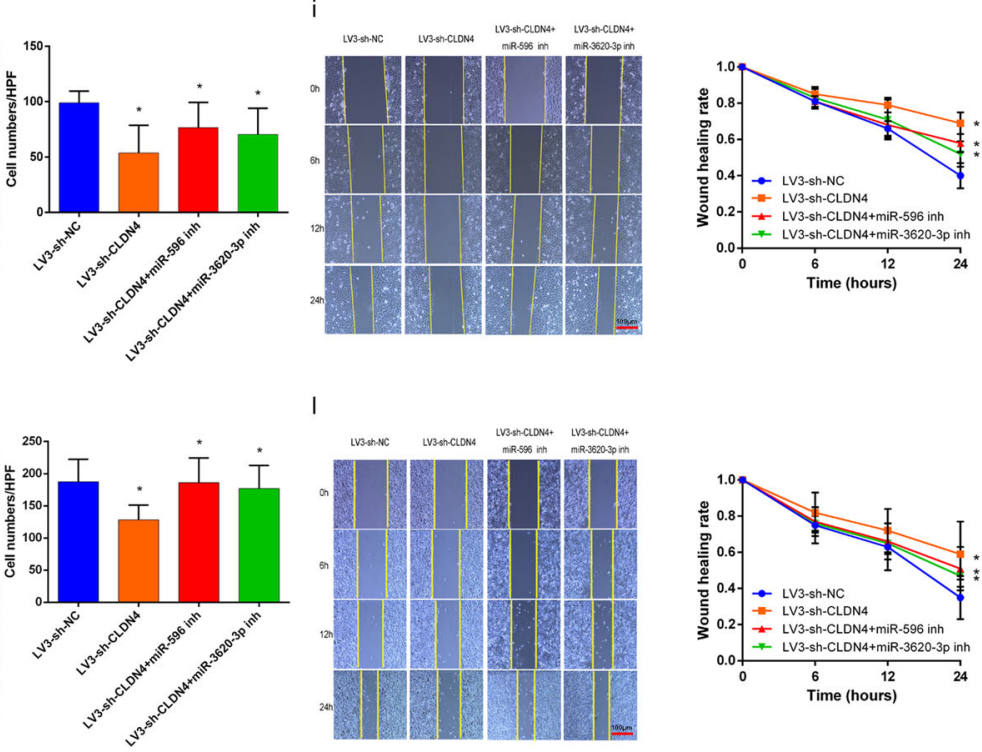

I
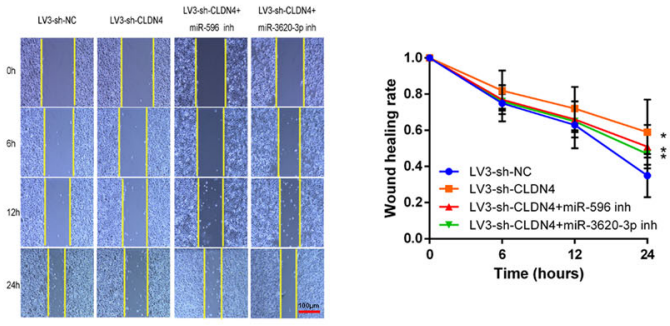

Published online: 19 May 2021

(c) (1) Open Access This article is licensed under a Creative Commons Attribution 4.0 International License, which permits use, sharing, adaptation, distribution and reproduction in any medium or format, as long as you give appropriate credit to the original author(s) and the source, provide a link to the Creative Commons license, and indicate if changes were made. The images or other third party material in this article are included in the article's Creative Commons license, unless indicated otherwise in a credit line to the material. If material is not included in the article's Creative Commons license and your intended use is not permitted by statutory regulation or exceeds the permitted use, you will need to obtain permission directly from the copyright holder. To view a copy of this license, visit http://creativecommons.org/licenses/by/4.0/.

(c) The Author(s) 2021 